\title{
Dislipidemia y Diabetes Mellitus tipo 2
}

\author{
Dr. Felipe Pollak ${ }^{1}$, Dr. Antonio Arteaga ${ }^{2}$ y Dra. Valentina Serrano ${ }^{3}$.
}

\section{Resumen}

A pesar de los evidentes avances en el tratamiento de la Diabetes Mellitus (DM), se ha observado en la últimas décadas un progresivo incremento de la patología cardiovascular en la DM tipo 2, hasta constituirse en su primera causa de muerte. No existen evidencias suficientes para atribuir este hecho solamente al nivel del control glucémico, destacando el posible rol de la resistencia insulínica y la mayor prevalencia de dislipidemia e hipertensión arterial.

Las dislipidemias constituyen una alteración común en la DM tipo 2, siendo la más común la llamada "dislipidemia aterogénica", elevación de los niveles de triglicéridos séricos, reducción del HDL-c y predominio de las partículas de $L D L$ pequeñas y densas. Pese a ello existen evidencias de que la utilización de estatinas reduce significativamente la incidencia de eventos cardiovasculares, por lo que constituyen la droga de elección, particularmente en prevención secundaria. Los fibratos, de mayor racionalidad por su mecanismo de acción, pero con evidencias de prevención menos consistentes, deben reservarse para pacientes con dislipidemia aterogénica en prevención primaria y en casos de hipertrigliceridemias severas para reducir el riesgo de pancreatitis.

\section{Summary}

In spite of the great advance on the treatment of Diabetes Mellitus (DM), cardiovascular mortality is progressively raising, and at the moment constitutes the first cause of death of DM type 2.

There are not enough evidences to claim blood glycemic control as the main cause of the high rates of cardiovascular morbid-mortality in DM type 2. Insulin resistance, common feature of DM type 2 is strongly suggested instead.

In this context, Dyslipidemia is highly prevalent. The common type is the so called "atherogenic dyslipidemia": high serum triglycerides, low HDL cholesterol and predominance of small, dense $L D L$ particles.

Statins are the drugs of choice, especially in secondary prevention. Fibrates can be used in primary prevention in presence of atherogenic dyslipidemia and in severe cases of hypertriglyceridemia in prevention of acute Pancreatitis.

At present treatment of DM type 2 must be intensive and multifactorial, reaching the recommended goals related to blood glucose, serum lipids and arterial pressure.

\section{Resumo}

Alem dos evidentes avances no enfrentamento da Diabetes Mellitus (DM), tem se observado nas últimas décadas um progressivo incremento da patologia cardiovascular na DM tipo 2, até se - constituir em sua primeira causa de morte. Não existem evidências suficientes para atribuir este fato somente ao nível de controle glicêmico, destacando a possível função da resistência insulínica e a maior prevalência de dislipidemia e hipertensão arterial. As dislipidemias constituem uma alteração comum na DM tipo 2, sendo a mais comum a chamada de "dislipidemia aterogênica", elevação dos níveis triglicerídeos séricos, redução do $H D L-c$ e predomínio das partículas de $L D L$ pequenas e densas. No entanto, existem evidências de que a utilização de estatinas reduz significativamente a incidência de eventos cardiovasculares, pelo que constituem a droga de eleição, particularmente em

\footnotetext{
Trabajo recibido el 26/01/07 y aceptado el 27/2/07

1 Profesor Auxiliar, Departamento de Nutrición, Diabetes y Metabolismo, Facultad de Medicina, Pontificia Universidad Católica de Chile

2 Profesor Emérito, Departamento de Nutrición, Diabetes y Metabolismo, Facultad de Medicina, Pontificia Universidad Católica de Chile.

${ }^{3}$ Departamento de Nutrición, Diabetes y Metabolismo, Facultad de Medicina, Pontificia Universidad Católica de Chile.
}

Dirección Postal: Dr. Felipe Pollak Cornejo. Departamento de Nutrición, Diabetes y Metabolismo. Facultad de Medicina, Pontificia Universidad Católica de Chile Lira 40, $4^{\circ}$ piso, Edificio de Gastroenterología. Santiago, Chile. Teléfono: 56-2-6863862 E-mail: fpollak@med.puc.cl 
prevenção secundária. Os fibratos, de maior racionalidade por seus mecanismos de ação, mas com evidências de prevenção menos consistentes, devem-se reservar para pacientes com dislipidemia aterogênica em prevenção primaria e nos casos de hipertrigliceridemias severas para reduzir o risco de pacreatite.

\section{Introducción}

La enfermedad cardiovascular constituye una complicación frecuente de la Diabetes Mellitus tipo 2 (DM 2). En la actualidad, dos tercios de los pacientes portadores de DM 2 fallece por Enfermedad Coronaria, Cerebrovascular o por una complicación asociada a Enfermedad Vascular Periférica ${ }^{(1,2,3)}$

En el "United Kingdom Prospective Diabetes Study", los diabéticos tipo 2 tratados de manera intensiva (Hemoglobina Glucosilada [ HbA1c ] de 7,0\%) presentaron una disminución de $16 \%$ de los eventos coronarios en comparación a los pacientes tratados en forma convencional (HbA1c de 7,8\%), diferencia estadísticamente no significativa ( $p=0,052$ ), lo que sugiere que un control estricto de la glicemia no es suficiente para la prevención cardiovascular( ${ }^{(4)}$

La elevada frecuencia de ateroesclerosis en la DM 2 se explica por varios factores:

1. Hipertensión arterial

2. Dislipidemia

3. Stress oxidativo

4. Anomalías de la fibrinolisis y de factores de coagulación

5. Hiperagregabilidad plaquetaria

6. Hiperhomocisteinemia

7. Disfunción endotelial

8. Malos hábitos de vida (por ej. tabaquismo)

El análisis de los resultados de trabajos prospectivos recientes, nos señala que la reducción de los niveles de lípidos séricos en los pacientes diabéticos, tanto en prevención primaria como secundaria, se traduce en una reducción significativa del riesgo de eventos cardiovasculares. Estos hallazgos son concordantes con el análisis del UKPDS, en el cual los niveles de Colesterol LDL (LDL-c) fue el principal indicador independiente de riesgo cardiovascular, lo que demuestra la importancia del óptimo manejo de la dislipidemia en estos pacientes ${ }^{(5)}$

En el siguiente artículo revisaremos la dislipidemia del paciente diabético tipo 2 y sus diferentes alternativas terapéuticas.

\section{Características de la dislipidemia}

Los trastornos lipídicos son un hallazgo frecuente en la DM 2. El estudio prospectivo de Framingham, con 30 años de seguimiento, demostró que los pacientes diabéticos presentan una incidencia significativa de hipertrigliceridemia y de niveles de Colesterol HDL (HDL-c) inferiores a $35 \mathrm{mg} / \mathrm{dl}$. También se observaron en mujeres, tasas de Colesterol total $>240 \mathrm{mg} / \mathrm{dl}$ en un $40 \%$ de los $\operatorname{casos}^{(6)}$. A partir de estos hallazgos podemos señalar que la dislipidemia habitual de un paciente diabético se caracteriza por una hipertrigliceridemia (en ayuno y post prandial), un nivel de HDL-c bajo, y niveles normales o moderadamente elevados de Colesterol total y LDL-c, esto último sobre todo en mujeres (7). Las partículas de LDL son pequeñas y densas, con un alto contenido en apoproteína B100 (apo B100) y una mayor capacidad aterogénica. Se ha descrito también, especialmente en presencia de nefropatía, un aumento de los niveles de lipoproteína (a), a la que se le atribuyen propiedades aterogénicas y pro-trombóticas ${ }^{(8)}$

\section{Etiopatogenia}

Existen varios factores involucrados en la etiopatogenia de las dislipidemias de la DM2, destacando aquellos relacionados con cambios directos de los efectos biológicos de la insulina en el metabolismo de las lipoproteínas Resistencia Insulínica (RI), otros derivados de la hiperglicemia, que a través de la glicosilación y peroxidación, de proteínas estructurales y de fase rápida, al cambiar la morfología y funcionalidad de ellas y de sus receptores y finalmente otras derivadas de la aparición de complicaciones específicas, como la nefropatía.

En la DM2 del sujeto obeso, aproximadamente un $80 \%$ presentan RI, y éste parece ser el mecanismo clave de la dislipidemia en estos pacientes. La secuencia se inicia por la incapacidad de la insulina en condiciones de RI de suprimir la actividad de la lipasa del tejido adiposo, lo que se asocia a una mayor lipólisis, y liberación de ácidos grasos y glicerol. La mayor concentración de ácidos grasos libres unidos a albúmina (NEFA) en el espacio vascular, incrementa su captación y transporte a nivel hepático y estimula su esterificación, lo que incrementa la síntesis y secreción de lipoproteínas de muy baja densidad (VLDL), ello se expresa en laboratorio clínico por un incremento de la concentración sérica de los niveles de triglicéridos. Existe gran controversia acerca de la actividad de la lipasa lipoproteica periférica (LLP) en DM2. Si bien la insulina es clave para la síntesis celular y translocasión de LLP hacia el endotelio, no existen evidencias de una menor actividad en DM2, postulándose como un hecho posible que en algunos casos la LLP se vea superada por la abundancia del substrato. Ello contrasta con el rol central del déficit de LLP, en la dislipidemia de la DM tipo 1.

Al existir un incremento de las concentraciones séricas de las lipoproteínas ricas en triglicéridos, se induce un mayor transporte de triglicéridos de éstas hacia HDL y LDL, por intermedio de la proteína transportadora de colesterol éster (CEPT), cuya actividad está aumentada 
en esta patología(9), enriqueciendo ambas partículas con triglicéridos. Existen evidencias que la afinidad de las lipasas por las distintas clases de lipoproteínas se incrementa en relación al contenido de triglicéridos de éstas. Así la actividad lipolítica de la lipasa lipoproteica hepática, se acentúa al recibir a las HDL y LDL enriquecidas en triglicéridos. Ello lleva a cambios estructurales y de composición de las LDL, que finalizan siendo más pequeñas y densas, con mayor susceptibilidad a la oxidación y menor afinidad al receptor de LDL. En cambio las HDL enriquecidas en triglicéridos al ser hidrolizadas por la lipasa lipoproteína hepática, sufren un proceso de disociación, con liberación de la fracción apoproteica, la cual es captada y excretada a nivel renal, lo que incrementa su catabolismo, traduciéndose en una reducción de los niveles de HDL-c.

Existen evidencias de la glucosilación y peroxidación de LDL y HDL, en DM2 descompensada, lo que está claramente relacionado con la hiperglucemia, ello acentúa la glucosilación de las apolipoproteinas y de las proteínas estructurales de los receptores, lo que contribuye básicamente a reducir el catabolismo tanto de las LDL y lipoproteínas ricas en triglicéridos ${ }^{(10)}$

Por otra parte, la complicación renal acentúa la RI y el síndrome nefrótico se asocia a una dislipidemia severa cuya etiopatogenia es poco clara, y que en las etapas avanzadas se expresa como una hipercolesterolemia severa, lo que es inhabitual en la DM 2 no complicada. Por último la elevada prevalencia de dislipidemias en la población general, obliga también a considerar en el paciente con DM2 la posibilidad de una asociación al azar. Lo que parece especialmente notorio en aquellos pacientes bebedores excesivos de alcohol o mujeres con terapia estrogénica anticonceptiva o de reemplazo hormonal.

\section{Reducción de lípidos y eventos cardiovascula- res en DM 2}

Los estudios prospectivos tanto en prevención primaria como secundaria, en pacientes diabéticos, tratados con fibratos o estatinas, demuestran que la reducción de los lípidos es fundamental para disminuir el riesgo de eventos cardiovasculares

Fibratos: en el estudio "Helsinski Heart Study", un subgrupo de 135 pacientes diabéticos tratados con gemfibrozilo mostraron una reducción del riesgo cardiovascular de un $68 \%$ (que no alcanzó a ser significativo dado el número reducido de pacientes)(11). La misma droga fue utilizada en el estudio "Veteran Affair High-density Lipoprotein Intervention Trial" (VA-HIT), que demostró una reducción del riesgo de un $24 \%$ $(p=0.05)$, en un subgrupo de 627 sujetos diabéticos con cardiopatía coronaria y HDL-c bajo(12)

Por otro lado, en el estudio de prevención secundaria "Diabetes Atherosclerosis Intervention Study" (DAIS), se siguieron 418 pacientes diabéticos, a los que se les randomizó a recibir fenofibrato o placebo durante 3 años, obteniéndose una reducción no significativa (23\%) para presentar un nuevo evento clínico y una reducción significativa en el porcentaje de progresión de la estenosis de arterias coronarias por angiografía(13)

Recientemente la publicación del estudio "Fenofibrate Intervention and Event Lowering in Diabetes" (FIELD), ha permitido comprender mejor el rol de los fibratos en la dislipidemia de los pacientes diabéticos. En este estudio se randomizaron 9.765 pacientes (7.664 en prevención primaria y 2.131 en prevención secundaria) los que recibieron fenofibrato o placebo por 5 años. El estudio no pudo demostrar una reducción significativa del "endpoint" primario cardiovascular. Sin embargo al hacer el análisis por subgrupos, el resultado fue positivo en prevención primaria, principalmente en presencia de HDL-c bajo (14)

Estatinas: si bien la elevación del LDL-c no es la alteración más frecuente en los pacientes diabéticos, las estatinas han sido las drogas que han demostrado mayores beneficios en el tratamiento de la dislipidemia (15). En el estudio "Cholesterol and Recurrent Clinical Events" (CARE), un subgrupo de 586 pacientes con antecedentes de enfermedad coronaria y colesterol total inferior a $240 \mathrm{mg} / \mathrm{dl}$, fue randomizado a pravastatina o placebo, obteniéndose en el grupo intervenido una reducción en el riesgo cardiovascular de $25 \%(p=0.05)^{(16)}$

En el estudio de prevención secundaria "Scandinavian Simvastatin Survival Study" (4S), un subgrupo de 202 diabéticos con hipercolesterolemia, tratados con simvastatina, presentaron una reducción de eventos de $55 \%(p=0.001)^{(17)}$

Si bien estos resultados confirman el beneficio del tratamiento hipolipemiante, el número reducido de pacientes en estos estudios y la ausencia de protocolos diseñados exclusivamente para pacientes diabéticos suscitaban algunos interrogantes.

Fue la publicación del estudio "Heart Protection Study" (HPS), la que aportó los elementos de respuesta. Durante 5 años, 5.963 pacientes diabéticos (aproximadamente $10 \%$ eran de tipo 1), recibieron 40 mgs. de simvastatina o placebo para obtener un LDL-c inferior a $115 \mathrm{mg} / \mathrm{dl}$. De este grupo 1.981 individuos tenían antecedentes de enfermedad coronaria o enfermedad vascular periférica. El uso de simvastatina permitió obtener una reducción de riesgo significativa de un $22 \%$ para un primer evento cardiovascular mayor. Este beneficio fue independiente de la edad, sexo, presión arterial, IMC, calidad del control glucémico, o LDL-c inicial (18)

Posteriormente el estudio "Collaborative Atorvastatin Diabetes Study" (CARDS), primer estudio específico para pacientes diabéticos en el cual ingresaron 2.838 pacientes considerados de alto riesgo (con hipertensión o retinopatía o albuminuria), recibieron atorvastatina 10 mgs. o placebo, independiente del valor de sus lípidos 
plasmáticos. Al cabo de 4 años, los sujetos que recibieron estatina presentaron una reducción en el riesgo relativo de un evento primario (muerte cardiovascular, infarto al miocardio, angina inestable, revascularización miocárdica y accidente vascular cerebral) de un 37\%. Al igual que en estudio HPS, los beneficios fueron independientes de las características de los pacientes y de su control glucémico ${ }^{(19)}$

\section{Objetivos Terapéuticos}

El principal objetivo del tratamiento, es obtener valores óptimos de LDL-c. Para pacientes con triglicéridos $>200 \mathrm{mg} / \mathrm{dl}$ se recomienda calcular el colesterol no HDL (no HDL-c):

$$
\text { no-HDL-c = Colesterol total - HDL-c }
$$

que representa el contenido de colesterol en las partículas de LDL, VLDL, y remanentes (todas aterogénicas ) y cuya meta terapéutica debe ser $30 \mathrm{mg} / \mathrm{dl}$ sobre el objetivo de LDL-c. En estos casos representa un objetivo secundario de nuestro tratamiento.

\section{Prevención primaria}

La recomendación actual de la NCEP ATP III y de la Asociación Americana de Diabetes (ADA) es considerar al paciente diabético de riesgo cardiovascular máximo, es decir, como equivalente coronario $(20,21)$

Los objetivos de tratamiento son:

- C-LDL $<100 \mathrm{mg} / \mathrm{dl}$,

- no - HDL- $C<130 \mathrm{mg} / \mathrm{dl}$,

- $\mathrm{C}-\mathrm{HDL}>40 \mathrm{mg} / \mathrm{dl}$,

- triglicéridos < $150 \mathrm{mg} / \mathrm{dl}$.

Según las normas europeas, en ausencia de otros factores de riesgo (como microalbuminuria), un nivel de LDL-c < $115 \mathrm{mg} / \mathrm{dl}$ puede ser considerado como aceptable(22)

Todo paciente que presente valores de lípidos sobre los objetivos mencionados debe ser considerado como dislipidémico.

\section{Prevención secundaria}

Los últimos estudios prospectivos (en particular el HPS) han establecido que existe una reducción del riesgo cardiovascular en los individuos diabéticos, hasta niveles de LDL-c por debajo de $70 \mathrm{mg} / \mathrm{dl}^{(23)}$. Considerando este aspecto, las recomendaciones para este grupo son:

- LDL-C deseable $<70 \mathrm{mg} / \mathrm{dl}$, aceptable $<100 \mathrm{mg} / \mathrm{dl}$,

- no-HDL-c deseable $<100 \mathrm{mg} / \mathrm{dl}$,

- HDL- c > $40 \mathrm{mg} / \mathrm{dl}$, aceptable $<130 \mathrm{mg} / \mathrm{dl}$,

- triglicéridos $<150 \mathrm{mg} / \mathrm{dl}$.
Estas recomendaciones fueron confirmadas en el estudio "Treating to New Targets" (TNT), que incluye un subgrupo de 1.501 pacientes diabéticos con enfermedad vascular que fueron randomizados a atorvastatina 10 mgs. u 80 mgs. para obtener niveles de LDL -c de 98 $\mathrm{mg} / \mathrm{dl}$ y $77 \mathrm{mg} / \mathrm{dl}$ respectivamente. Fueron seguidos por 5 años, en los que el grupo tratado en forma intensiva presentó una reducción significativa del riesgo relativo para un evento vascular mayor de un $25 \%$ (1\% de reducción de riesgo por cada $1 \mathrm{mg} / \mathrm{dl}$ de LDL-c) $)^{(24)}$

\section{Tratamiento de la dislipidemia}

Se recomienda iniciar manejo no farmacológico, en presencia de C-LDL entre 100 y 130 mg/dl, durante unos 3 a 6 meses. Si el objetivo no se consigue en ese periodo debe indicarse un medicamento hipolipemiante. Por otra parte, debe iniciarse de inmediato manejo farmacológico en presencia de enfermedad vascular o con LDL-c mayor a $130 \mathrm{mg} / \mathrm{dl}$.

Medidas terapéuticas no farmacológicas: corresponden fundamentalmente a: un control glucémico estricto, medidas dietéticas y actividad física regular.

Control glucémico: se ha demostrado que un control glucémico estricto (optimización de Hba1c), puede reducir significativamente los niveles de lípidos sanguíneos. En diabéticos descompensados que presentan hipertrigliceridemia (menor a $500 \mathrm{mg} / \mathrm{dl}$ ), es posible normalizar los niveles de triglicéridos únicamente corrigiendo la glucemia plasmática(25)

Medidas dietéticas: tienen un efecto beneficioso al disminuir la insulinoresistencia, reducir la glucemia y mejorar el perfil lipídico. En casos de exceso ponderal, se recomiendan 20 calorías por $\mathrm{Kg}$. de peso ideal, para promover la pérdida de peso. Si existe un estado de eutrofia, el aporte debe ser normocalórico. Los hidratos de carbono deben corresponder al $45-60 \%$ del aporte calórico, principalmente de tipo complejo, y limitando la sacarosa. No se recomienda aportar edulcorantes calóricos (fructosa o sorbitol). El aporte de lípidos no debe superar el 30\% de las calorías y su distribución debe ser la siguiente:

- Grasas saturadas y trans $<7 \%$

- Grasas poliinsaturadas $10 \%$

- Grasas monoinsaturadas > 10\%

El aporte de colesterol no debe superar los 200 mgs. al día y la ingesta de fibra debe ser de 20 a 30 grs diarios. Debe restringirse el aporte de alcohol en individuos obesos e hipertrigliceridémicos por su aporte calórico y ya que aumenta la producción hepática de VLDL $(20,26)$

Actividad física: favorece la baja de peso, aumenta la sensibilidad a la insulina a nivel muscular y mejora los 
niveles de HDL-c. Se recomiendan entre 30 y 45 minutos de ejercicio ligero a moderado (caminar, ciclismo, natación, otros deportes recreativos), al menos 3 veces por semana. En los pacientes coronarios, la actividad física debe ser programada según recomendación del cardiólogo.

Estas medidas han demostrado eficacia en la corrección de la hipertrigliceridemia, aumentar moderadamente el HDL-c , y en reducir el LDL-c entre un 10 y $20 \%$.

\section{Tratamiento farmacológico}

\section{No específico:}

Los pacientes diabéticos con Síndrome Metabólico se benefician del uso de drogas insulinosensibilizantes. Con el uso de metformina y tiazolidinedionas (rosiglitazona y pioglitazona), efectivos reductores de insulinoresisitencia, es posible obtener una leve mejoría en el perfil lipídico en grados variables, además de beneficios sobre otros factores de riesgo (por ej., niveles de presión arterial). También se puede obtener una reducción en la proporción de partículas de LDL pequeñas y densas y un aumento de las de mayor tamaño, que son de menor

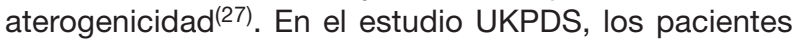
obesos que recibieron metformina presentaron una reducción significativa de eventos coronarios en comparación con el grupo tratado de manera convencional(28). Finalmente, el estudio de prevención secundaria "Prospective Pioglitazone Clinical Trial in Macrovascular Events" (PROACTIVE) demostró que la pioglitazona reduce el riesgo de presentar un evento clínico cardiovascular en un $16 \%$ (diferencia no significativa)(29) $^{(29)}$

\section{Específico (hipolipemiantes):}

La elección del hipolipemiante depende del tipo de dislipidemia y debe estar basada en evidencia clínica.

a. Hipercolesterolemia aislada (LDL-c elevado, triglicéridos normales): las estatinas son el medicamento de elección. Estudios prospectivos randomizados, han demostrado que reducen significativamente los eventos coronarios en prevención primaria y secundaria ${ }^{(15)}$. Si los lípidos séricos no se normalizan con la monoterapia, debe asociarse un segundo hipolipemiante. La elección debe ser ezetimibe o una resina (por ej., colesteramina, la que debe indicarse con prudencia ya que puede elevar los niveles de triglicéridos).

b. Hipertrigliceridemia aislada (triglicéridos elevados, no HDL-c y LDL-c normales): los fibratos son el medicamento de elección. Con triglicéridos sobre $500 \mathrm{mg} / \mathrm{dl}$ predomina el riesgo de pancreatitis aguda. El gemfibrozilo es el más efectivo y eleva el HDL-c significativamente. En caso de no responder a monoterapia se recomienda asociar a acido nicotínico (o un derivado como el acipimox) o bien a acidos grasos Omega 3. c. Dislipidemia mixta (LDL-c y triglicéridos sobre los objetivos): es la situación más frecuente. La estatina es el medicamento de primera elección. Deben utilizarse las de mayor potencia por su mayor efecto en triglicéridos (simvastatina, atorvastatina o rosuvastatina). En pacientes con LDL-c menor a 130 $\mathrm{mg} / \mathrm{dl}$, y sólo en prevención primaria, puede usarse un fibrato de $2^{\circ}$ generación (fenofibrato, ciprofibrato, bezafibrato) como monoterapia.

En caso de necesitar asociación pueden considerarse las siguientes alternativas:

estatina + acido nicotínico (o acipimox ): en el estudio HATS ("HDL-Atherosclerosis Treatment Study") que incluyó 160 pacientes en prevención secundaria, 16\% diabéticos, esta asociación aumentó el HDL-c en un $26 \%$ y redujo los eventos cardiovasculares dramáticamente ${ }^{(30)}$. Existe riesgo de miopatía, pero en menor medida que en la asociación con fibrato. El acido nicotínico puede inducir rubor facial, el que se puede prevenir tomando aspirina 1 hora antes del medicamento.

estatina + fibrato: es muy eficaz, pero aumenta el riesgo de miopatía en relación a la monoterapia. En un estudio reciente, 40 pacientes con DM 2 y dislipidemia mixta fueron tratados con atorvastatina más fenofibrato, cumpliéndose los objetivos de la ADA para LDL-c en un $97,5 \%$, para triglicéridos en un $100 \%$, y en un $60 \%$ para HDL-c. No se observaron casos de rabdomiolísis y los efectos adversos fueron semejantes a la monotera$\mathrm{pia}^{(31)}$. La asociación está contraindicada en pacientes con insuficiencia renal, polifarmacia y en ancianos. En la asociación debe usarse un fibrato de $2^{\circ}$ generación ya que el gemfibrozilo aumenta significativamente el riesgo de miopatía en asociación y es un pobre reductor de LDL-c ${ }^{(32)}$

Estatina + acidos grasos Omega 3: estos últimos deben utilizarse en dosis de 4 a 6 grs al día. No se han descrito efectos adversos.

\section{Intervención multifactorial sobre factores de riesgo}

El tratamiento de la dislipidemia es sólo parte de una estrategia de prevención cardiovascular que debe incluir:

1. Control estricto de glucemia

2. Normalización de presión arterial

3. Utilización de antiagregantes plaquetarios

4. Reducción de peso y aumento de la actividad física

5. Suspensión del tabaco

La eficacia de un tratamiento multifactorial fue demostrada por Gaede y cols en el estudio "Steno 2", en el cual se randomizaron 160 pacientes diabéticos tipo 2 con microalbuminuria, a un tratamiento convencional o intensivo. Se definió como tratamiento intensivo un objetivo de $\mathrm{HbA} 1 \mathrm{c}<6,5 \%$, presión arterial $<130 / 80 \mathrm{mmHg}$, 
colesterol total $<175 \mathrm{mg} / \mathrm{dl}$ y triglicéridos $<150 \mathrm{mg} / \mathrm{dl}$. Un $85 \%$ de los pacientes recibió estatinas. Se recomendó actividad física liviana (30 minutos 3 a 5 veces a la semana), reducción de peso y suspensión del tabaco. A los 8 años de seguimiento, se encontró una significativa reducción en la aparición y progresión de nefropatía, retinopatía y neuropatía autonómica en el grupo tratado intensivamente. Se obtuvo además una reducción de un $53 \%$ ( $p=0,007)$ del riesgo de eventos vasculares mayores, lo que representa la mayor disminución de eventos obtenida en estudios prospectivos para este tipo de pacientes ${ }^{(33)}$

\section{Otros efectos beneficiosos de los hipolipemiantes}

Otro aspecto importante de mencionar son los efectos extralipídicos beneficiosos de las estatinas. En el estu- dio WOSCOPS ("West of Scotland Coronary Prevention Study") la utilización de pravastatina redujo en un $30 \%$ el riesgo de presentar DM $2(p=0,042)^{(34)}$. Esta observación ha sido atribuida a los efectos pleiotrópicos y antiinflamatorios de las estatinas, en los mediadores de insulinoresistencia como IL-6 y TNF $\alpha$.

Por otra parte, un metanálisis indica que la utilización de estatinas disminuye la proteinuria y protege la filtración glomerular en los pacientes con enfermedad renal crónica, lo que fue confirmado en el estudio HPS, en el que los pacientes asignados a placebo presentaron un mayor deterioro de su función renal (niveles de creatinina) en comparación al grupo asignado a simvastatina(35)

Finalmente en el estudio FIELD, el fenofibrato redujo significativamente la progresión de la albuminuria y la indicación de fotocoagulación en la retinopatía.

Los mecanismos de estos hallazgos no han sido aclarados y deberán ser confirmados por futuros estudios.

\section{Bibliografía}

1. Haffner S, Letho S, Ronnemaa T, Pyörälä $\mathrm{K}$, Laakso M. Mortality from coronary heart disease in subjects with type 2. Diabetes and in non diabetic subjects with prior myocardial infarction. N.Engl.J.Med. 1998; 339: 229.234

2. Whiteley L, Padmanabhan S, Hole D, Isles C. ¿Should Diabetes be considered a Coronary Heart Disease Risk Equivalent? Results from 25 years of follow-up in the Renfrew and Paisley Survey. Diabetes Care. 28: 1588-93, 2005

3. Juutilainen A, Lehto S, Rönnemaa T, Pyörälä K, Laakso M. Type 2 Diabetes as a Coronary Heart Disease Equivalent. A 18-year prospective population-based study in Finnish subjects. Diabetes Care. 28 : 2901-07, 2005

4. UK Prospective Diabetes Study (UKPDS) Group. Intensive blood glucose control with sulphonylureas or insulin compared with conventional treatment and risk of complications in patients with type 2 Diabetes. Lancet. 1998; 317: 703-711.

5. Turner RC, Millns H, Neil HA, et al. Risk factors for coronary artery disease in non-insulin dependent diabetes mellitus: United Kingdom Prospective Diabetes Study (UKPDS: 23). BMJ. 1998, Mar 14; 316(7134): 823-8

6. Siegel RD, Cupples A, Schaefer EJ, Wilson PW. Lipoproteins, apolipoproteins and low-density lipoprotein size among diabetics in the Framingham offspring study. Metabolism. 1996, Oct; 45(10): 1267-72

7. Steiner G. Dyslipoproteinemias of Diabetes.Atherosclerosis. 1994; 110 ( suppl.): S27-33

8. Ziegler O, Guerci B, Candiloros H, Drouin P. Lipoprotein (a) and diabetes mellitus. Diabetes Metab. 1995 Apr; 21 (2): 127-38

9. de Vries R, Perton FG, Dallinga-Thie GM, et al. Plasma cholesteryl ester transfer is a determinant of intima-media thickness in type 2 diabetic and non diabetic subjects: role of CETP and triglycerides. Diabetes. 2005, Dec; 54(12): 3554-9
10. Kolovou GD, Anagnostopoulou KK, Cokkinos DV. Pathophysiology of dyslipidemia in the metabolic syndrome.Postgrad Med 2005; 81: 358-366.

11. Koskinen $P$, Manttari M, Manninen V, Huttunen JK, Heinonen OP, Frick MH. Coronary heart disease incidence in NIDDM patients in the Helsinki Heart Study. Diabetes Care 1992 Jul; 15(7):820-5.

12. Rubins HB, Robins SJ, Collins D, et al. Gemfibrozil for the secondary prevention of coronary heart disease in men with low levels of high density lipoprotein cholesterol. Veteran's Affair High Density Lipoprotein Cholesterol Intervention Trial (VA-HIT) Study Group. N. Engl. Med. 1999; 341: 410-418.

13. Steiner G, and the Diabetes Atherosclerosis Intervention Study (DAIS). Effect of Fenofibrate on progression of coronary artery disease in type 2 diabetes. A randomized study. Lancet. 2001; 357: 905-910

14. The FIELD study investigators. Effects of long-term fenofibrate therapy on cardiovascular events in 9765 people with type 2 diabetes mellitus (the FIELD study): randomized controlled trial. Lancet 2005; 366: 1849-61

15. Costa J, Borges M, David C, Vaz Carneiro A. Efficacy of lipid lowering drug treatment for diabetic and nondiabetic patients: meta-analysis of randomised trials. BMJ. 2006; 332(7550): 1115-24.

16. Goldberg R, Mellies M, Sacks F, et al. Cardiovascular events and their reduction with Pravastatin in diabetic and glucose intolerant myocardial infarction survivors with average cholesterol levels: subgroup analysis in The Cholesterol and Recurrent Events (CARE) trial. Circulation. 1998; 98: 2513-2519

17. Pyorala K, Pedersen Tr, Kjekhus J, Faergeman $\mathrm{O}$, Olsson AG, Thorgeirsson G. Cholesterol lowering with Simvastatin improves prognosis of diabetic patients with coronary heart disease: A subgroup analysis of the $4 \mathrm{~S}$ study. Diabetes Care. 1997; 20: 614-620

18. Collins R, Armitage J, Parish S, Sleigh P, Peto R, 
and the Heart Protection Study Collaborative Group. MRC/BHF Heart Protection Study of cholesterol-lowering with Simvastatin in 5963 people with diabetes: a randomised placebo-controlled trial. Lancet. 2003; 361: 2005-2014

19. Colhoun HM, Betteridge DJ, Durrington PN, et al (CARDS study investigators). Primary prevention of cardiovascular disease with Atorvastatin in type 2 Diabetes in the Collaborative Atorvastatin Diabetes Study (CARDS): multicentre randomized placebo-controlled trial. Lancet 2004; 364: 685-96

20. Third report of the National Cholesterol Education Program (NCEP) on the detection, evaluation and treatment of high blood cholesterol in adult (ATP III). Executive summary. NIH publication 01-3670, May 2001 (www. nhlbi.nih.gov/guidelines/cholesterol/)

21. American Diabetes Association. Management of dyslipidemia in adults with Diabetes. Diabetes Care. 2003; 26 (suppl.1): S83-86

22. Debacker G. European guidelines on cardiovascular disease prevention in clinical practice. Eur J Cardiov Prev Rehab. 2003; 10: S1-S78.

23. Grundy S, Cleeman J, Merz $\mathbf{C N}$, et al, for the Coordinating Committee of the National Cholesterol Education program. Implications of recent Clinical Trials for the National Education Program Adult Treatment Panel III Guidelines. Circulation 2004; 110: 227-239.

24. Sheperd J, Barter P, Carmena R, et al, for the Treating to new Targets Investigators. Effect of lowering LDL Cholesterol substantially below currently recommended levels in patients with Coronary Heart Disease and Diabetes. The Treating to New Targets ( TNT) study. Diabetes Care 2006; 29: 1220-26

25. Dunn FL. Management of hyperlipidemia on diabetes mellitus. Endocrinol Metab Clin North Am. 1992 Jun;21(2): 395-414

26. American Diabetes Association. Standars of Medical care in Diabetes. Diabetes Care, 28 (Suppl.1), January 2005: S4-36
27. Buse JB, Tan MH, Prince MJ, Erickson PP. The effects of oral anti-hyperglycemic medications on serum lipid profiles in patients with type 2 diabetes. Diabetes Obes Metab. 2004 Mar; 6(2): 133-56

28. UK Prospective Diabetes Study Group. Effect of intensive blood-glucose control with metformin on complications in overweight patients with type 2 diabetes (UKPDS 34). Lancet. 1998 Sep 12; 352(9131): 854-65

29. Dormandy JA, Charbonnel B, Eckland DJ, et al (PROactive investigators). Secondary prevention of macrovascular events in patients with type 2 diabetes in the PROactive Study (PROspective pioglitAzone Clinical Trial in macroVascular events): a randomized controlled trial. Lancet 2005; 366: 1279-89

30. Brown BG, Zhao XQ, Chait A, et al. Simvastatin and Niacin, antioxidant vitamins, or the combination for the prevention of Coronary Disease. N Engl J Med. 2001; 345: 1538-39

31. Athyros VG, Papageorgiou AA., Athyrou VV., Demitriadis DS., Kontopoulos AG. Atorvastatin and micronized Fenofibrate alone and in combination in type 2 diabetes with combined hyperlipidemia. Diabetes Care. 2002; 25: 1198-1202

32. Malcolm Law, Alicja R. Rudnicka. Statin Safety: A Systematic Review. Am Journal of Cardiology. 2006, April; 97(8), Suppl.1: S52-60

33. Gaede P., Vedel P, Larse N, Jensen GV, Parving HH, Pedersen O. Multifactorial intervention and cardiovascular disease in patients with type 2 diabetes. N.Engl.J.Med. 2003; 248: 383-393

34. Freeman D, Norrie J, Sattar N, et al. Pravastatin and the development of Diabetes Mellitus. Evidence for a protective treatment effect in the West of Scotland Coronary Prevention Study. Circulation 2001; 103: 357362

35. Douglas K, O'Malley PG, Jackson JL. Meta-analysis: the effect of statins on albuminuria. Ann Intern Med. 2006 Jul 18;145 (2):117-24. 